\title{
The role of cerebellar impairment in emotion processing: a case study
}

\author{
Alexandra K Gold and Rosemary Toomey ${ }^{*}$ (D)
}

\begin{abstract}
Background: Though the cerebellum's role in visuospatial and fine motor functioning has been well-established over the last several years, the role of the cerebellum in emotion has more recently been a focus of scientific inquiry. Cerebellar impairment has been associated with deficits in emotional processing and is linked to a wide range of clinical behaviors including social withdrawal, blunted emotional expression, and impulsivity. In addition, cerebellar impairments have been associated with the onset of psychiatric disorders including major depressive disorder and, more recently, obsessive-compulsive disorder.

Case presentation: We describe a 32-year-old patient who presented to our clinic for a neuropsychological evaluation with a childhood history of a cerebellar brain tumor and detail-oriented, perfectionistic tendencies. Neuropsychological assessment data revealed impairments in visuospatial processing and in fine motor skills, likely stemming from the cerebellar tumor. Clinical assessment led to a diagnosis of obsessive-compulsive personality disorder and also suggested impairments in socio-emotional processing.

Conclusions: Our findings lend support to recent data which has suggested the impact of cerebellar impairment on emotional processing and related domains. Unlike many previous studies, however, our report focuses on an individual who, despite having marked impairments in certain domains, demonstrates a high level of functioning. We believe that this report holds important clinical relevance for proper diagnosis of cerebellar-related impairment and for the necessity of early intervention.
\end{abstract}

Keywords: Cerebellum, Emotion, Obsessive-compulsive personality disorder

\section{Background}

Given the cerebellum's implicated role in motor control and spatial navigation, a vast literature has linked cerebellar impairments to reduced visuospatial capacities and loss of fine motor functioning [1-5]. Over the past several years, many researchers have begun to explore the cerebellum's role across varied domains of emotion function including emotional recognition, emotional learning, and emotional processing [6, 7]. Findings on the contributions of the cerebellum to an array of emotion domains have been observed in healthy and clinical populations. One recent study in healthy individuals found that applying transcranial magnetic stimulation over the left cerebellum (e.g., portion of cerebellum thought to be involved in emotional processing) affected participants' abilities to accurately label emotional expressions and to specify the gender of emotional faces

\footnotetext{
* Correspondence: toomey@bu.edu

Department of Psychological and Brain Sciences, Boston University, 900 Commonwealth Avenue, 2nd Floor, Boston, MA, USA
}

whereas neutral expressions and faces were unaffected [8]. Clinically, uncharacteristic emotional features resulting from cerebellar damage have been noted such as disinhibited behavioral responses and impulsivity in a patient with a right cerebellar infarction [9], stubbornness, thought rigidity, and inappropriate behavior (e.g., indecent humor) in patients with right-sided cerebellar lesions [7], and blunted emotional affect, social withdrawal, and low motivation in patients with left-sided cerebellar lesions [7]. In an extension of these clinical data, one study inferred that patients with cerebellar lesions may have a poor comprehension of their own emotional state because they did not report mood symptoms that they were observed to exhibit [10]. Some data suggest that cerebellar damage occurring earlier in life may result in more readily apparent emotion deficits by impacting normative growth processes such as social development and communication [11]. Notably, emotional and behavioral deficits resulting from cerebellar 
impairments may stem from excessive or restricted responses to one's internal or external surroundings [12].

The emotional changes associated with cerebellar impairment have also been linked to the onset of several psychiatric conditions including depressive disorders, obsessive-compulsive disorder (OCD), and borderline personality disorder [12-15]. Whereas the association between cerebellar damage and major depression is well-documented $[13,16-19]$, only recently have studies begun to posit OCD pathology as stemming from impairments to the cerebellum. Within the past four years, case studies of patients with late-onset OCD hypothesize that left cerebellar lesions and cerebellar volumetric deficits may be implicating factors in OCD pathology $[12,20]$. In this case presentation, we describe a patient with a childhood cerebellar tumor who presented to our clinic for neuropsychological evaluation. Her symptom presentation was reflective of deficits in socio-emotional processing and obsessive-compulsive tendencies.

\section{Case presentation}

\section{Clinical history}

Patient was a 32-year-old Caucasian female graduate student who self-referred to the Boston University Center for Anxiety and Related Disorders for a neuropsychological evaluation in order to renew the academic accommodations that she reported having for the entirety of her education. These accommodations included having assistance with academic work requiring the use of fine motor skills, having access to someone who could take notes for her (e.g., to limit the amount of writing she needed to complete), being in a distraction-free environment, and having extra time for assignments. Prior to beginning assessment procedures, the patient reviewed and signed the informed consent form, which was approved by the Boston University Institutional Review Board. Patient reported that her need for academic accommodations stemmed from a childhood cerebellar tumor which necessitated several associated procedures from the ages of 4-12. After the first appearance of the cerebellar tumor when the patient was 4 years of age, the patient was required to undergo an 8 -h surgery to remove the tumor. Post-surgery hydrocephalus necessitated the placement of a ventral peritoneal shunt. Following this initial surgical procedure, the patient had right-sided paralysis, was wheelchair-bound for several months, and diagnosed with right hemiparesis, requiring her to become left-handed (e.g., in line with data suggesting cerebellar impairment can necessitate a switched handedness [21]). The tumor grew back when the patient was 8 years of age, leading to one subsequent procedure to remove the tumor (which also necessitated additional surgeries for shunt repairs and strabismus up until the patient was 12 years of age). At our assessment, the patient reported an unremitting impact of the tumor on her fine motor skills and on her ability to maintain good balance. Nonetheless, the patient reported a high level of academic success across her educational experiences. Despite noting that homework assignments took longer for her to complete than might be expected, she reported consistently receiving very superior scores on her coursework and that she had achieved high grade point averages in her secondary and graduate academic experiences.

Patient reported taking $20 \mathrm{mg}$ of Celexa for the past four years for OCD and symptoms of depression. Patient stated that she had been experiencing symptoms of OCD since childhood, having been diagnosed with the condition by her neurologist when she was 8 years old. Of note, the patient reported that OCD runs in her father's side of the family. Patient described her OCD as involving her brain "going on repeat," "getting caught on things," or "looping back" such that she was easily distracted by noises in her environment and had difficult focusing, leading her to continually check back and re-read every sentence that she would write. Patient reported experiencing "self-diagnosed anhedonia" since she was a college student though she noted that she had grown accustomed to these symptoms of low mood, stating that they did not interfere with her life. Patient also reported having non-diagnosed misophonia (e.g., strong reactions to particular sounds) since childhood. Patient had not recently received psychotherapy for these symptoms though she did see a pediatric psychologist beginning at age 5 and continuing until she was 9 years old. She also briefly received cognitive-behavioral therapy (CBT) for her OCD symptoms while in college though noted that she did not find the treatment to be particularly helpful. Patient stated that she performed ongoing "selfCBT" for her OCD, enabling her to function.

Patient reported having an "Asperger-like personality," contributing to feelings of isolation that were consistently present in her life. Patient did not have many friends growing up and she reported not having any close social relationships. She attributed her lack of interest in socializing to the long time that it took her to complete tasks, leading her to feel a constant time pressure to accomplish the tasks that were most important to her. Nonetheless, patient considered herself to be socially adept despite not being interested in seeking social relationships. She reported learning several tricks to blend in and have normal surface-level interactions; these included asking others about themselves, having her body face another individual when they were communicating, and applying self-deprecating humor in conversation.

In her prior neuropsychological report, the patient was diagnosed with Axis I Learning Disorder, Not Otherwise Specified (NOS) (Nonverbal Learning Disability [NVLD]) and OCD. The NVLD diagnosis was largely based on the discrepancy between the patient's verbal and spatial intellectual capacities as assessed by the Wechsler Adult 
Intelligence Scale-III (WAIS-III). OCD was not formally evaluated during the assessment process and was based on the patient's prior diagnosis and on behavioral observations collected during the assessment process.

\section{Neuropsychological assessments}

Table 1 depicts the outcomes for all neuropsychological assessments conducted during the patient's four testing sessions at Boston University. Standard scores (also referred to as scaled scores) are included throughout Table 1 and represent conversions of the raw scores onto a common scale. The distribution of standard scores have a mean of 100 and a standard deviation of 15 . We conducted assessments of intelligence and general achievement (e.g., Wechsler Adult Intelligence Scale-IV [WAIS-IV] [22], Woodcock Johnson IV Tests of Achievement [WJ-IV]) [23], social cognition (e.g., Advanced Clinical Solutions for the WAIS-IV [ACS]) [24], attention and executive functioning (e.g., Stroop test [25], Rey-Osterrieth Complex Figure Test [26, 27], Conners Continuous Performance Test 3 [CPT 3] [28], Delis-Kaplan Executive Function System Test [D-KEFS]) [29], memory (e.g., California Verbal Learning Test II [CVLT-II] [30], Wechsler Memory Scale-IV [WMS-IV]) [31], and motor functioning (e.g., Finger Tapping Test [32, 33], Grooved Pegboard Test [34]). For the purposes of this case report, we will focus on data from certain assessments.

Patient's performance on the WAIS-IV suggested a high level of general intellectual functioning with a Full-Scale Intelligence Quotient (FSIQ) in the 93th percentile, reflecting her overall superior cognitive capacities; of note, however, there was marked variability in her performance across domains of verbal comprehension, perceptual reasoning, working memory, and processing speed that comprise the FSIQ. Across the administered neuropsychological tests, patient's performance in the domain of verbal reasoning and utilization of verbal content emerged as a personal strength, with the patient scoring in the 99.9th percentile on the Verbal Comprehension Index.

Patient's performance on certain tests was consistent with impairments in visuospatial reasoning and fine motor skills, likely stemming from her cerebellar brain tumor. She exhibited her lowest WAIS-IV performance in the perceptual reasoning domain, scoring in the 34th percentile, though there was variability in the patient's performance across the various subtests that comprise the domain. For instance, she exhibited average performance on the Block Design, Visual Puzzles, and Matrix Reasoning subtests (with her score on Block Design being superior to her performance on the former two subtests) and very superior performance on the Figure Weights subtest. Assessments supporting the patient's visuospatial deficits included the WMS-IV Spatial Addition task (standard score of 3), WMS-IV Visual Reproduction tasks (particularly in correctly recognizing the originally presented figures, 3-9 percentile) and the Rey-Osterrieth Complex Figure Task ( $\leq 1$ percentile in copy, immediate recall, and delayed recall). Several tests also confirmed the patient's deficits in motor dexterity including the Finger Tapping Test and the Grooved Pegboard Test. On the Finger Tapping Test, across 5 trials on each hand, the patient exhibited approximately 14 taps over a 10-s duration; as a comparison, the age-matched normative sample mean across both hands ranges from approximately 45 taps to 49 taps. On the Grooved Pegboard Test, the patient exhibited several peg drops and, when using her left hand, completed the task in nearly $3 \mathrm{~min}$; this was markedly slower than the normative, age-matched sample mean task duration of approximately $1 \mathrm{~min}$. Of note, the patient's low performance on the Rey-Osterrieth Complex Figure Task also may have served as an indication of fine motor deficits as the patient had some difficulty in drawing straight, non-interrupted lines while copying the original image. Overall, neuropsychological testing data supported the impact of the patient's childhood cerebellar tumor on her present-day motor skills and visuospatial capabilities, warranting a diagnosis of major neurocognitive disorder due to another medical condition, with behavioral disturbance.

The patient's perfectionistic, detail-oriented tendencies were apparent across several assessments. Most notable was the patient's execution of the D-KEFS Trail Making Test Motor Speed Task which required her to trace a dotted line that connects several open circles as quickly as possible. During the practice trial, the patient asked the assessor whether she was supposed to connect each dash individually (e.g., from one dash to the next). The assessor informed the patient that she should instead focus on drawing the line as quickly as possible and not on connecting each individual dash. On the test round, the patient did not connect each dash individually but still completed the task in over $87 \mathrm{~s}$ (scaled score of 1), suggesting that her heightened focus on precision carried into the test administration. Other assessments revealed a pattern of over-responding such that the patient was "doing more" than she needed to for that particular task. For instance, on the CVLT-II, the patient demonstrated a high 16 repetitions during her recall across the 5 trials, reflecting her potential concern about missing a word. On the Conners CPT 3, the patient demonstrated a very elevated commission rate (e.g., responding to non-target letters), suggesting that she was worried about missing a letter as it flashed across the screen, thus leading her to over-respond to irrelevant stimuli. At other times, patient's perfectionistic tendencies manifested as her focusing so closely on the details of tasks such that she lost sight of their intended meaning. On the WAIS-IV Picture Completion Task, when asked to identify the missing elements of the image, the patient's responses suggested that she was focusing very closely on the fine details of the image and thus not able to 
Table 1 Neurocognitive test results

\begin{tabular}{|c|c|c|c|c|}
\hline \multirow[t]{2}{*}{ Neurocognitive test } & \multirow[t]{2}{*}{ Score } & \multirow[t]{2}{*}{ Population Mean (SD) } & \multicolumn{2}{|c|}{ Clinical Significance ${ }^{a}$} \\
\hline & & & $\geq-1 S D$ & $\geq-2 S D$ \\
\hline \multicolumn{5}{|l|}{ WAIS-IV } \\
\hline Verbal Comprehension Index & 149 & $100(15)$ & & \\
\hline Similarities & 19 & $10(3)$ & & \\
\hline Vocabulary & 19 & $10(3)$ & & \\
\hline Information & 16 & $10(3)$ & & \\
\hline Perceptual Reasoning Index & 94 & $100(15)$ & & \\
\hline Block Design & 11 & $10(3)$ & & \\
\hline Matrix Reasoning & 8 & $10(3)$ & & \\
\hline Visual Puzzles & 8 & $10(3)$ & & \\
\hline Figure Weights & 16 & $10(3)$ & & \\
\hline Picture Completion & 7 & $10(3)$ & $\sqrt{ }$ & \\
\hline Working Memory Index & 125 & $100(15)$ & & \\
\hline Digit Span & 14 & $10(3)$ & & \\
\hline Arithmetic & 15 & $10(3)$ & & \\
\hline Processing Speed Index & 105 & $100(15)$ & & \\
\hline Symbol Search & 12 & $10(3)$ & & \\
\hline Coding & 10 & $10(3)$ & & \\
\hline Cancellation & 2 & $10(3)$ & & $\sqrt{ }$ \\
\hline Full Scale IQ & 122 & $100(15)$ & & \\
\hline \multicolumn{5}{|l|}{ WAIS-IV ACS } \\
\hline Affect Naming & 5 & $10(3)$ & & $\sqrt{ }$ \\
\hline Faces I & 8 & $10(3)$ & & \\
\hline Faces \| & 13 & $10(3)$ & & \\
\hline \multicolumn{5}{|l|}{ WJ-IV } \\
\hline Passage Comprehension & 106 & $100(15)$ & & \\
\hline Calculation & 117 & $100(15)$ & & \\
\hline Sentence Reading Fluency & 116 & $100(15)$ & & \\
\hline Math Facts Fluency & 116 & $100(15)$ & & \\
\hline Sentence Writing Fluency & 103 & $100(15)$ & & \\
\hline Reading Recall & 118 & $100(15)$ & & \\
\hline Reading Vocabulary & 130 & $100(15)$ & & \\
\hline \multicolumn{5}{|l|}{ Stroop Test } \\
\hline Word & 25 & $50(10)$ & & $\sqrt{ }$ \\
\hline Color & 33 & $50(10)$ & $\sqrt{ }$ & \\
\hline Color-Word & 60 & $50(10)$ & & \\
\hline Interference & 61 & $50(10)$ & & \\
\hline \multicolumn{5}{|c|}{ Rey-Osterrieth Complex Figure Test } \\
\hline Copy & $\leq 1$ & Percentile & & $\sqrt{ }$ \\
\hline Immediate Recall & $\leq 1$ & Percentile & & $\sqrt{ }$ \\
\hline Delayed Recall & $\leq 1$ & Percentile & & $\sqrt{ }$ \\
\hline Organization & 53 & $50(10)$ & & \\
\hline Recognition Total Correct & 54 & $50(10)$ & & \\
\hline
\end{tabular}


Table 1 Neurocognitive test results (Continued)

\begin{tabular}{|c|c|c|c|c|}
\hline \multirow[t]{2}{*}{ Neurocognitive test } & \multirow[t]{2}{*}{ Score } & \multirow[t]{2}{*}{ Population Mean (SD) } & \multicolumn{2}{|c|}{ Clinical Significance ${ }^{a}$} \\
\hline & & & $\geq-1 S D$ & $\geq-2 S D$ \\
\hline \multicolumn{5}{|l|}{ CPT-3 } \\
\hline Response Style & 17 & $50(10)$ & & $\sqrt{ }$ \\
\hline Detectability & 58 & $50(10)$ & & \\
\hline$\%$ Omissions & 45 & $50(10)$ & & \\
\hline$\%$ Commissions & 76 & $50(10)$ & & $\sqrt{ }$ \\
\hline Perseverations & 48 & $50(10)$ & & \\
\hline Hit Reaction Time & 39 & $50(10)$ & $\sqrt{ }$ & \\
\hline Hit Reaction Time Standard Deviation & 46 & $50(10)$ & & \\
\hline Variability & 47 & $50(10)$ & & \\
\hline Hit Reaction Time Block Change & 57 & $50(10)$ & & \\
\hline Hit Reaction Time Interstimulus Interval Change & 40 & $50(10)$ & & \\
\hline \multicolumn{5}{|l|}{ D-KEFS Trail Making Test } \\
\hline Visual Scanning & 11 & $10(3)$ & & \\
\hline Number Sequencing & 7 & $10(3)$ & $\sqrt{ }$ & \\
\hline Letter Sequencing & 8 & $10(3)$ & & \\
\hline Number Letter Switching & 11 & $10(3)$ & & \\
\hline Motor Speed & 1 & $10(3)$ & & $\sqrt{ }$ \\
\hline \multicolumn{5}{|l|}{ CVLT-II } \\
\hline Total Hits Trials 1-5 & 55 & $50(10)$ & & \\
\hline Short Delay Free Recall & 1.5 & $0(1)$ & & \\
\hline Long Delay Free Recall & 1 & $0(1)$ & & \\
\hline Recognition & $100 \%$ & Percent Recognized & & \\
\hline \multicolumn{5}{|l|}{ WMS-IV } \\
\hline Symbol Span & 13 & $10(3)$ & & \\
\hline Spatial Addition & 3 & $10(3)$ & & $\sqrt{ }$ \\
\hline Visual Reproduction I & 12 & $10(3)$ & & \\
\hline Visual Reproduction II & 8 & $10(3)$ & & \\
\hline Visual Reproduction Recognition & $3-9$ & Percentile & $\sqrt{ }$ & \\
\hline \multicolumn{5}{|l|}{ Finger Tapping Test ${ }^{\mathrm{b}}$} \\
\hline Left hand (taps) & 14.4 & $M=49.0, S D=4.1$ & & $\sqrt{ }$ \\
\hline Right hand (taps) & 14.8 & $M=44.6, S D=4.6$ & & $\sqrt{ }$ \\
\hline \multicolumn{5}{|l|}{ Grooved Pegboard ${ }^{b}$} \\
\hline Left hand (seconds) & 105 & $M=69.66, S D=19.27$ & $\sqrt{ }$ & \\
\hline Right hand (seconds) & 180 & $M=75.80, S D=21.56$ & & $\sqrt{ }$ \\
\hline
\end{tabular}

WAIS-IV Wechsler Adult Intelligence Scale-IV [22]; WJ-IV Woodcock Johnson IV Tests of Achievement [23], ACS Advanced Clinical Solutions for the WAIS-IV [24], CPT 3 Conners Continuous Performance Test 3 [28], D-KEFS Delis-Kaplan Executive Function System Test [29], CVLT-II California Verbal Learning Test II [30], WMS-IV Wechsler Memory Scale-IV [31]

${ }^{\text {a }}$ Reflects clinically relevant results at two thresholds: greater than or equal to either one or two standard deviations more impaired than the mean. Scores that represent better performance than the mean are not indicated. CPT-3 Hit RT can be clinically significant in either direction from the mean (lower than mean is faster performance)

b Indicated means and SDs are from standardization samples

adopt a broader perspective in determining the most essential missing component. For instance, when asked to identify what was missing in an image depicting a hand holding a rose, she stated that "the person is not grasping the rose tightly enough." When she was asked what was missing in an image of a snowy landscape that featured a barn, the patient reported that barn doors typically have a second element on their door that allows the door to be opened or closed, as opposed to identifying the more essential missing piece. 
We conducted a test of social cognition to provide further context for the patient's self-reported "Asperger-like" personality and the patient's prior NVLD diagnosis. The WAIS-IV ACS Affect Naming Test required the patient to correctly label the emotion being expressed by a target face from a list of seven possible emotions (happy, sad, angry, afraid, surprised, disgusted, and neutral). Out of 24 identification questions, the patient identified 9 incorrectly; this performance corresponded to the 5th percentile. Notably, 4 incorrect identifications involved the patient not recognizing when a face was "afraid" and 3 of these incorrect identifications involved the patient labeling a face as "angry" when the correct answer was "no feeling/neutral." The ACS Faces Test involved cards featuring various faces, some of which were placed on a grid in front of the patient for several seconds. Once the face cards were removed, the patient was asked to select the previously presented cards and place them in the correct spots on the grid. Unlike the ACS Affect Naming Test, the ACS Faces Test did not involve emotion recognition. The patient performed in the average range on the ACS Faces Test immediate recall (Faces I; 25th percentile) and in the high average range on delayed recall (Faces II; 84th percentile).

\section{Clinical assessments and interview}

We conducted two structured clinical assessments in order to gather additional clinical data relevant to the patient's self-reported anhedonia and previously-diagnosed OCD. The Anxiety and Related Disorders Interview Schedule for DSM-5 (ADIS-5) [35] is a structured interview that evaluates current mood, anxiety, obsessive-compulsive, trauma, and related disorders. Upon formal assessment, patient did not meet criteria for OCD (endorsing neither compulsions nor obsessions) or major depressive disorder/ persistent depressive disorder (with her only salient symptom in this domain being depressed mood). The Structured Clinical Interview for DSM-IV Axis II Personality Disorders (SCID-II) [36] is a structured assessment that provides a formal evaluation of personality disorders by inquiring on specific diagnostic criteria. Given the patient's history of perfectionistic, obsessive-compulsive tendencies, we formally assessed for obsessive-compulsive personality disorder (OCPD). During this assessment, the patient reported that she was the kind of person to focus on order, organization, and details, noting that she liked to make schedules and lists. Patient reported having a mental checklist for her school work, her hygiene, and her food (e.g., ensuring that she followed a specific calorie count and ate certain nutrients). Patient reported sometimes getting so caught up in her focus on these areas that she lost sight of what she was trying to accomplish; she provided the example of putting on sunscreen in the morning and being very focused on not missing a spot such that she would realize after 20 min that she was still putting sunscreen on her face. She also reported having trouble finishing jobs "all the time" because she was focused on getting things exactly right and not missing anything; for instance, on an exam, she would spend a great deal of time on her responses to make sure that she did not make any mistakes, leading a test to take much longer than it would have if she had simply followed her instincts. Patient reported being so focused on certain domains of her life - specifically, her schoolwork, food consumption, hygiene, and regular exercise routine - such that she did not have much remaining time for pursuing friendships. Patient also reported experiencing some difficulty in discarding non-disposable items, largely attributed to the patient's concern about having to buy something again at a later point, leading her apartment to be very cluttered as a result. Patient reflected that, as a child, she was very stubborn, had strong opinions, and talked back frequently. Overall, given the patient's responses, we provided the patient with an OCPD diagnosis.

\section{Discussion and conclusions}

This case study explores a patient presenting to our clinic with a childhood history of a cerebellar tumor that had contributed to an ongoing pattern of visuospatial impairments and fine motor skill deficits. Other patterns of the clinical presentation - specifically, the patient's detail-oriented, perfectionistic tendencies, her self-described anhedonia, and her socio-emotional impairments - were less readily accounted for by her complex medical history. However, emerging data associating cerebellar damage with restricted socio-emotional processing and the onset of psychiatric conditions such as OCD, depressive disorders, and personality pathology may provide some additional insight on this case and on subsequent similar presentations. Indeed, this presentation extends the existing literature on this topic in many ways. First, whereas most prior case studies have generally focused on patients with current and/or recent acute cerebellar conditions in adulthood [7, 9], this case study centered on a patient with a childhood history of a cerebellar tumor and thus provides some insight on the ongoing impact of cerebellar damage even years after the initial medical event. Of note, one recent case study reported the onset of borderline personality disorder in a patient following the rupture of a cerebellum arteriovenous malformation that had been present since the patient's childhood [15]. Though the lesion in this study occurred in adulthood, the presence of cerebellar abnormality in the patient's youth is somewhat comparable to the present case study. Moreover, prior case studies have generally centered on individuals whose cerebellar impairment has resulted in overall cognitive slowing $[7,10]$. Whereas the presented patient does have marked deficits in motor control and visuospatial processing, her superior cognitive and general intellectual 
functioning (e.g., WAIS-IV FSIQ in the 93rd percentile, $\mathrm{VCI}$ in the 99.9th percentile) is notable as is her self-reported high-level of academic achievement. Further, to our knowledge, our study is among the first to report on the potential association between cerebellar impairment and the onset of OCPD; of note, early signs of the patient's detail-oriented, perfectionistic tendencies were present in her childhood, manifesting at the time as stubbornness (e.g., talking back to her mom) and thus providing further support for the possible role of the patient's cerebellar tumor in her OCPD pathology. Notably, the link between cerebellar impairment and OCPD is in line with prior case studies which have noted increased rigidity of thought and stubbornness in patients with right-sided cerebellar lesions [7]. It should be noted that we did not have access to the patient's medical records, thus precluding us from providing additional diagnostic information about the patient's cerebellar tumor and associated procedures. The one other relevant case study that enrolled a patient with OCPD reported on an individual with a left cerebellar lesion who had a premorbid diagnosis of OCPD and late-onset OCD with the latter condition hypothesized to be linked to the cerebellar lesion given the patient's age (e.g., as OCD after 50 generally stems from an organic basis [37]) [12]. Specifically, the authors suggested that the OCD may have developed from an interaction between the lack of "homeostatic control" created by the cerebellar lesion and the patient's already-existent OCPD [12]. In contrast, the patient that we discuss in this case report was diagnosed with OCD when she was 8 years old, around the time of her second cerebellar tumor surgery, for a pattern of behaviors that included perseveration, excessive hand washing, and working on math problems for a long time even after she had already solved the problem. Such symptoms are reflective of the types of detail-oriented, perfectionistic tendencies that we noted in the patient throughout our assessment process, yet the patient did not meet current OCD diagnostic criteria. OCD is marked by the presence of obsessions (e.g., recurrent, persistent thoughts, images, or urges that the individual attempts to neutralize or suppress) or compulsions (e.g., repetitive behaviors or mental acts that the individual feels motivated to complete in response to an obsession) [38], neither of which the patient endorsed. OCPD is rooted in a preoccupation with perfectionism and orderliness, often while sacrificing efficiency and flexibility [38]; this pattern was reflective of the patient's symptoms and thus informed our diagnostic choice of OCPD.

Further, despite the patient's social skill deficits which are often seen in patients with NVLD [38], we felt that the patient's visuo-spatial and fine motor deficits were better accounted for by an organic basis (e.g., her childhood brain tumor), thus leading us to instead diagnose her with a major neurocognitive disorder due to another medical condition. We added the "with behavioral disturbance" specifier to account for her impairments across other domains of functioning (e.g., social processing deficits). Our confidence in this diagnosis was enhanced by the fact that the patient's previous NVLD diagnosis had been presumably entirely based on the discrepancy between her verbal and spatial abilities on the WAIS-III.

Our assessment process thus resulted in the changing of two diagnoses - 1) NVLD to major neurocognitive disorder due to another medical condition, with behavioral disturbance and 2) OCD to OCPD. The necessity of these diagnostic changes highlights the importance of exercising great caution when assigning diagnoses to patients with these types of complex neurological histories (e.g., cerebellar tumors) as symptoms can be overlapping across diagnostic conditions, making differentiation a challenge. For instance, it is not unusual for a patient to have comorbid OCD and OCPD with $23-32 \%$ of OCD patients also having a comorbid diagnosis of OCPD [12, 39]. Thus, it is possible that the symptoms of the one diagnosis could blend into the symptoms of the other diagnosis, potentially leading to misdiagnosis of a given patient's condition. Proper diagnosis is essential so that a patient can be directed down an appropriate path of treatment, as opposed to a path that may not be necessary and/or advisable.

The relatively high comorbidity of OCD and OCPD suggest a possible link between these two conditions $[12,39,40]$. Given that the patient had a family history of OCD, it is possible that her past OCD and current OCPD presentations were compounded by her having learned modeled behavior and/or her having a genetic vulnerability. However, in light of the recent literature linking cerebellar impairment with emotion deficits and OCD pathology $[12,20]$, the patient's illness presentation suggests the role of factors that extend beyond familial psychiatric history $[7,9,10,12]$.

At the conclusion of our assessment process, we provided the patient with specific recommendations for academic accommodations and subsequent clinical care. The patient's marked deficits in visuospatial reasoning and fine motor skill domains warranted provision of continued academic accommodations including access to someone who could take notes for her, assistance with filling in the bubbles on multiple-choice tests, double time on assessments, restricted amount of writing and typing that she needed to complete, and presentation of visual information in a verbal format whenever possible. We felt that the patient's perfectionistic, detail-oriented tendencies likely had an overall adverse impact on her task efficiency and cognitive flexibility, leading us to refer her for CBT at our clinic to help her better understand her perfectionistic inclinations and thus reduce their impact on her cognitive performance. We also noted that CBT would likely be useful for helping 
the patient with her ongoing, self-identified symptoms of anhedonia and in supplying her with social skills training that could enhance the quality of her relationships with others.

Our case study provides a glimpse into the clinical presentation of a high-functioning individual with a childhood brain tumor associated with characteristic present-day deficits (e.g., impaired visuospatial processing and poor fine motor control) and a unique array of additional clinical characteristics (e.g., detail-oriented, perfectionistic tendencies, self-reported anhedonia, blunted socio-emotional processing). Despite the patient's superior level of overall daily functioning, her marked adult impairments in emotional and social processing suggest that a childhood cerebellar tumor may leave an imprint across multiple domains. Thus, our findings suggest the importance of early, intensive psychotherapy, social skills training, and cognitive rehabilitation as possible preventive measures in the immediate aftermath of cerebellar damage.

\section{Abbreviations}

ACS: Advanced Clinical Solutions for the WAIS-IV; ADIS-5: Anxiety and Related Disorders Interview Schedule for DSM-5; CPT 3: Conners Continuous Performance Test 3; CVLT-II: California Verbal Learning Test II; D-KEFS: DelisKaplan Executive Function System Test; SCID-II: Structured Clinical Interview for DSM-IV Axis II Personality Disorders; WAIS-IV: Wechsler Adult Intelligence Scale-IV; WJ-IV: Woodcock Johnson IV Tests of Achievement; WMS-IV: Wechsle Memory Scale-IV

\section{Availability of data and materials}

The data that support the findings of this study are potentially available on request from the corresponding author (R.T.) and following participant permission. The data are not publicly available so as to protect the participant's confidentiality.

\section{Authors' contributions}

AKG was responsible for coordinating and executing the assessments. RT was responsible for design of the assessment process and for the training and supervision of AKG on all aspects of the case. Both authors contributed to the preparation and writing of the manuscript.

\section{Ethics approval and consent to participate}

Upon visiting the Boston University Center for Anxiety and Related Disorders, patients sign a Consent for Neuropsychological Assessment that includes a statement providing permission for archival or retrospective research. The Boston University Charles River Campus Institutional Review Board has approved the use of a Neuropsychological Clinical Research Data Repository associated with this clinical service.

\section{Consent for publication}

The participant provided her written consent for the creation of this manuscript and approved the submitted version of the manuscript.

\section{Competing interests}

The authors declare that they have no competing interests.

\section{Publisher's Note}

Springer Nature remains neutral with regard to jurisdictional claims in published maps and institutional affiliations.
Received: 12 June 2018 Accepted: 28 September 2018

Published online: 12 October 2018

\section{References}

1. Molinari M, Petrosini L, Misciagna S, Leggio M. Visuospatial abilities in cerebellar disorders. J Neurol Neurosurg Psychiatry. 2004;75(2):235-40.

2. Levisohn L, Cronin-Golomb A, Schmahmann JD. Neuropsychologica consequences of cerebellar tumour resection in children: cerebellar cognitive affective syndrome in a paediatric population. Brain. 2000;123(5):1041-50.

3. O'Halloran CJ, Kinsella GJ, Storey E. The cerebellum and neuropsychological functioning: a critical review. J Clin Exp Neuropsychol. 2012;34(1):35-56.

4. Bastian AJ. Moving, sensing and learning with cerebellar damage. Curr Opin Neurobiol. 2011;21(4):596-601.

5. Millichap J. Motor Deficits after Cerebellar Tumor Resection. Pediatric Neurology Briefs. 2005;19(6):41-2.

6. Adamaszek M, D'agata F, Ferrucci R, Habas C, Keulen S, Kirkby K, et al. Consensus paper: cerebellum and emotion. Cerebellum. 2017;16(2):552-76.

7. Siuda K, Chrobak AA, Starowicz-Filip A, Tereszko A, Dudek D. Emotional disorders in patients with cerebellar damage--case studies. Psychiatr Pol. 2014:48(2):289-97.

8. Ferrari C, Oldrati V, Gallucci M, Vecchi T, Cattaneo Z. The role of the cerebellum in explicit and incidental processing of facial emotional expressions: a study with transcranial magnetic stimulation. Neurolmage. 2018;169:256-64.

9. van Dun K, De Witte E, Van Daele W, Van Hecke W, Manto M, Mariën P. Atypical cerebral and cerebellar language organisation: a case study. Cerebellum ataxias. 2015;2(1):18.

10. Bolceková E, Mojzeš M, Van Tran Q, Kukal J, Ostrý S, Kulišták P, et al. Cognitive impairment in cerebellar lesions: a logit model based on neuropsychological testing. Cerebellum ataxias. 2017;4(1):13.

11. Wang SS, Kloth AD, Badura A. The cerebellum, sensitive periods, and autism Neuron. 2014;83(3):518-32

12. Tonna M, Ottoni R, Ossola P, De Panfilis C, Marchesi C. Late-onset obsessivecompulsive disorder associated with left cerebellar lesion. Cerebellum 2014;13(4):531-535

13. Phillips JR, Hewedi DH, Eissa AM, Moustafa AA. The cerebellum and psychiatric disorders. Front Public Health. 2015;3:66.

14. Schmahmann JD, Weilburg JB, Sherman JC. The neuropsychiatry of the cerebellum -insights from the clinic. Cerebellum. 2007;6(3):254-67.

15. Lupo M, Olivito G, Siciliano L, Masciullo M, Bozzali M, Molinari M, et al. Development of a psychiatric disorder linked to cerebellar lesions. Cerebellum. 2018:1-9.

16. Liu L, Zeng L-L, Li Y, Ma Q, Li B, Shen H, et al. Altered cerebellar functional connectivity with intrinsic connectivity networks in adults with major depressive disorder. PLoS One. 2012;7(6):e39516.

17. $M a$ Q, Zeng $L-L$, Shen $H$, Liu L, Hu D. Altered cerebellar-cerebral restingstate functional connectivity reliably identifies major depressive disorder. Brain Res. 2013;1495:86-94.

18. Peng J, Liu J, Nie B, Li Y, Shan B, Wang G, et al. Cerebral and cerebellar gray matter reduction in first-episode patients with major depressive disorder: a voxel-based morphometry study. Eur J Radiol. 2011:80(2):395-9.

19. Minichino A, Bersani FS, Trabucchi G, Albano G, Primavera M, Delle Chiaie R, et al. The role of cerebellum in unipolar and bipolar depression: a review of the main neurobiological findings. Rivista di psichiatria. 2014;49(3):124-31.

20. Narayanaswamy JC, Jose D, Kalmady SV, Agarwal SM, Venkatasubramanian G, Reddy YJ. Cerebellar volume deficits in medication-naïve obsessive compulsive disorder. Psychiatry Res Neuroimaging. 2016;254:164-8.

21. Mascalchi M, Lenge M, Bianchi A, Bartolini E, Gavazzi G, Giordano F, et al. Hemicerebellitis can drive handedness shift. Cerebellum ataxias. 2017:4(1):14.

22. Wechsler D. Wechsler adult intelligence scale-fourth edition (WAIS-IV). San Antonio, Texas: Psychological Corporation; 2014.

23. Schrank FA, McGrew KS, Mather N, Wendling BJ, LaForte EM. WoodcockJohnson IV Tests of achievement: woodcock-Johnson IV tests of cognitive abilities. Rolling Meadows: Riverside publishing company; 2014.

24. Pearson N. Advanced clinical solutions for WAIS-IV and WMS-IV: administration and scoring manual. San Antonio: The Psychological Corporation; 2009.

25. Golden C, Freshwater S. Stroop color and word test, revised 2002 adult manual for clinical and experimental uses. Wood Dale, IL: Stoelting; 2002.

26. Rey A. L'examen psychologique dans les cas d'encéphalopathie traumatique.(Les problems.). Archives de psychologie. 1941. 
27. Osterrieth PA. Le test de copie d'une figure complexe; contribution à l'étude de la perception et de la mémoire. Archives de psychologie. 1944.

28. Conners C. Conners continuous performance test 3. North Tonawanda, NY: Multi-Health Systems Inc.; 2014

29. Delis DC, Kramer JH, Kaplan E, Ober, BA. California Verbal Learning TestSecond Edition. San Antonio: The Psychological Corporation; 2000.

30. Delis DC, Kramer JH, Kaplan E, Ober B. California verbal learning test II (CVLT II). 2nd ed. San Antonio, TX: The Psychological Corporation; 2000.

31. Wechsler D. Wechsler memory scale - fourth edition (WMS-IV) administration and scoring manual. San Antonio,TX: Pearson; 2009.

32. Halstead W. Brain and intelligence: university press; 1947.

33. Resources PA. The finger tapping test. Florida: Lutz; 1992.

34. Company LI. The grooved pegboard test. Indiana: Lafayette; 2002.

35. Brown TA, Barlow DH. Anxiety and related disorders interview schedule for DSM-5, adult and lifetime version: clinician manual. New York: Oxford University press; 2013.

36. First MB, Benjamin LS, Gibbon M, Spitzer RL, Williams JB. Structured clinical interview for DSM-IV Axis II personality disorders. Washington D.C.: American psychiatric press; 1997.

37. Kumar V, Chakrabarti S, Modi M, Sahoo M. Late-onset obsessive compulsive disorder associated with possible gliomatosis cerebri. World J Biol Psychiatry. 2009;10(4 Pt 2):636-9.

38. APA. Diagnostic and statistical manual of mental disorders. 5th ed. Arlington, VA: American Psychiatric Association; 2013.

39. Ecker W, Kupfer J, Gonner S. Incompleteness as a link between obsessivecompulsive personality traits and specific symptom dimensions of obsessivecompulsive disorder. Clinical psychol psychother. 2014;21(5):394-402.

40. Starcevic V, Berle D, Brakoulias V, Sammut P, Moses K, Milicevic D, et al. Obsessive-compulsive personality disorder co-occurring with obsessivecompulsive disorder: conceptual and clinical implications. Aust N Z J Psychiatry. 2013;47(1):65-73.

Ready to submit your research? Choose BMC and benefit from:

- fast, convenient online submission

- thorough peer review by experienced researchers in your field

- rapid publication on acceptance

- support for research data, including large and complex data types

- gold Open Access which fosters wider collaboration and increased citations

- maximum visibility for your research: over $100 \mathrm{M}$ website views per year

At $\mathrm{BMC}$, research is always in progress.

Learn more biomedcentral.com/submissions 